\title{
Omega-3 Fatty Acid Ethyl-Eicosapentaenoate Attenuates IL-I $\beta$-Induced Changes in Dopamine and Metabolites in the Shell of the Nucleus Accumbens: Involved with PLA2 Activity and Corticosterone Secretion
}

\author{
Cai Song*, ${ }^{*, 2,3}$, Xuwen $\mathrm{Li}^{3}$, Zhijian $\mathrm{Kang}^{3}$ and Yoshie Kadotomi ${ }^{3}$ \\ 'Department of Biomedical Sciences, AVC, University of Prince Edward Island, Charlottetown, PE, Canada; ${ }^{2}$ Canada National Institute for \\ Nutrisciences and Health, Charlottetown, PE, Canada; ${ }^{3}$ Department of Psychiatry, University of British Columbia, Vancouver, BC, Canada
}

\begin{abstract}
Previously, we have reported that interleukin-I $\beta$ (IL-I) induces changes in dopaminergic (DA) and serotonergic systems in the core of nucleus accumbens (NAc). We have also demonstrated that $n-3$ fatty acid ethyl-eicosapentaenoate (EPA) can significantly reduce stress and anxiety-like behaviors, corticosterone concentration and peripheral inflammatory response induced by $\mathrm{IL}-\mathrm{I}$ administration. Compared to the core, the shell is involved more in emotion, stress and psychiatric diseases. However, the relationship between inflammation and the functions of DA system in the shell has not been studied. Since phospholipase (PL) $A 2$ is a key enzyme in arachidonic acid (AA)- eicosanoids-prostaglandin (PG)E2 pathway, and the change in NAc dopaminergic system has been associated with glucocorticoid stimulation, therefore, the hypotheses of this study are (I) that IL-I induced changes in DA neurotransmission in the shell may be through PLA2-PGE2-corticosterone pathway; (2) EPA may attenuate IL-I effects via inhibiting PLA2 activities, which blocks PGE2 stimulation of corticosterone. Using an in vivo microdialysis method, the present study showed that IL-I administration significantly increased extracellular levels of DA, and its metabolites 3,4-dihydroxyphenylacetic acid (DOPAC) and homovanillic acid (HVA) in the shell of the NAc. IL-I also increased blood concentration of corticosterone and PGE2, and increased the activities of cytosolic and sectory of PLA2. IL-I-induced changes were significantly attenuated by EPA treatment. Furthermore, glucorcoticoid receptor antagonist mifepristone (RU486) pretreatment significantly blocked IL-I-induced changes in DA and metabolites. Qquinacrine, a PLA2 antagonist significantly blocked IL-I induced increase in PGE2 and corticosterone concentrations. These results demonstrated the hypotheses that IL-I effects may be via PLA2-PGE2-corticosterone pathway and EPA attenuated IL-I effects may be through the suppression of PLA2 expression, which then reduced PGE2 synthesis and corticosterone secretion.

Neuropsychopharmacology (2007) 32, 736-744. doi: I 0.1038/sj.npp. I 30 I I I7; published online I4 June 2006
\end{abstract}

Keywords: dopamine; nucleus accumbens; IL- I $\beta$; n-3 fatty acid EPA; PLA2 enzymes; RU486

\section{INTRODUCTION}

Recent studies have indicated that various types of stress activate dopaminergic and serotonergic systems in mesocortical and mesolimbic areas of the brain, which are related to mood disorder (Heimer et al, 1997; Fulford and Marsden, 1998a, b; Noguchi et al, 2001). The nucleus accumbens (NAc) is an important part of the mesolimbic dopamine (DA) system. In the NAc, especially in the shell, changes in

*Correspondence: Dr C Song, Department of Biomedical Sciences, AVC, University of Prince Edward Island and NRC Institute for Nutrisciences and Health, 93 Mount Edward Road, Charlottetown, PE, Canada CIA 5TI, Tel: + I 902566 7977, Fax: + I 902569 4289,

E-mail: cai.song@nrc.gc.ca

Received 18 October 2005; revised 26 April 2006; accepted I May 2006

Online publication: II May 2006 at http://www.acnp.org/citations/ Npp05 I | 06050634/default.pdf
DA and its metabolites occur in response to variety of stressor including an immune challenge (Kalivas and Duffy, 1995; Wu et al, 1999; Bland et al, 2003; Delarue et al, 2003). Systemic administration of proinflammatory cytokine interleukin-1 $\beta$ (IL-1) has been reported to reduce libido and social interaction, stimulate glucocorticoid secretion, induce anhedonia and stress/anxiety-like behavior (Dantzer et al, 1998; van der Meer et al, 1996; Brebner et al, 2000; Anisman et al, 2002). Previous microdialysis studies have shown that systemic administration of IL-1 significantly increased the extracellular concentrations of HVA and 5HIAA in the core of the NAc, which was exacerbated by a stressor application (Fulford and Marsden, 1998b, Song et al, 1999). Compared to the NAc core, the shell is involved more in motivation, reward, stress response and psychiatric disorders (Altman et al, 1996; Egan and Weinberger, 1997; Nestler et al, 2002). In addition, stress-induced changes in dopaminergic and serotonergic systems in the NAc shell 
have been associated with the glucocorticoid secretion (Barrot et al, 2000; Piazza et al, 1996). However, the possible mechanism by which proinflammatory cytokines-caused change in neurotransmission and behavior has not been studied in the shell of the NAc.

It is known that the effects of IL-1 on brain functions are mediated by prostaglandin (PG)E2 via the activation of the phospholipase A2-arachidonic acid-cyclooxygenase2 pathway after immune stimuli (Engblom et al, 2002). Cytosolic (c) and secretory (s) phospholipase A2 (PLA2) are well known to involve in neuroinflammation because they can trigger the release of n-6 fatty acid arachidonic acid (AA), a precursor for synthesis of eicosanoids (Sun et al, 2004; Phillis and O'Regan, 2004; Rosenberger et al, 2004), which can produce PGE2 and proinflammatory cytokines. PGE2 binding to neurons in the ventrolateral medulla activates corticotrophin-releasing factor (CRF)-containing neurons in the paraventricular hypothalamic nucleus (PVN), which elicits corticotrophin release (Engblom et al, 2002). A increase in accumbal and hypothalamic AA concentrations have been reported in an animal model of depression, which has been postulated to contribute to the dysfunction of the DA system and some depressive behavior (Green et al, 2005). Thus, a proposed pathway by which IL-1 may induce changes in NAc DA system is shown in Figure 1.

Previously, we have reported that polyunsaturated omega (n)-3 fatty acid, eicosapentaenoic acid (EPA), attenuated IL-1-induced stress and anxiety-like behavior, and reduced IL-1-induced secretion of prostaglandin (PG) E2 and corticosterone (Song et al, 2003; Song and Horrobin, 2004). EPA has also been shown to reduce PLA2 expression in patients with autistic spectrum disorders (Bell et al, 2004). Thus, n-3 fatty acids may compete the inflammatory effects of n-6 fatty acid AA and inhibit the changes in neurotransmitter and neuroendocrine systems induced by inflammation (Figure 1). Therefore, the hypotheses of the present study are: (1) systemic IL-1 administration may increase hypothalamic cPLA2 and sPLA2 activities that then increase PGE2 and corticosterone synthesis, which can be attenuated by a PLA2 inhibitor; (2) increased corticosterone following IL-1 administration may induce changes in DA and metabolites in the shell, which can be blocked by glucocorticoid receptor antagonist; (3) n-3 fatty acid EPA can attenuate IL-1 effects by reducing PLA2 activities, PGE2 and corticosterone secretion. To demonstrate these hypotheses: (1) the effect of IL-1 and EPA on the activity of CPLA2 (liberates AA in the brain) (Diez et al, 1992), sPLA2-IIA (plays a role in several types of brain injury and inflammation) (Phillis and O'Regan, 2004) and PGE2 were measured in the hypothalamus, and (2) the extracellular concentration of DA and its metabolites in the shell were measured by an in vivo microdialysis technique and HPLC following saline or IL-1 administration in rats fed control or EPA diet; (3) the relationship between cortico- sterone secretion and accumbal DA and metabolites were studied by pretreatment of glucocorticoid receptor antagonist mifepristone (RU486) before IL-1 or saline administration in animals; (4) the effect of EPA on IL-1-induced stress hormone corticosterone was measured in the serum and (5) effects of PLA2 inhibitor on IL-1-induced changes in PGE2 and corticosterone were also determined.

\section{MATERIALS AND METHODS}

\section{Experimental Design}

Animal body weight was measured once a week before the surgery, and daily after surgery or after IL-1 injection for a week.

In experiment 1, the effect of EPA on systemic IL-1induced changes in DA and its metabolites were studied in the shell of the NAc. Animals were divided into four groups of 10 rats. Two groups of animals were fed normal rat chow supplemented with $1 \%$ palm oil as a control diet, and the other two groups of animals were fed rat chow supplemented with 1\% EPA. Each diet group was treated with saline and IL-1 (i.p.) respectively. After surgery for the implantation of a guide cannula at the shell of the NAc, animals were allowed to recover for 2 weeks. Rats were then transferred to individual testing cage and allowed to acclimatize for 2 days prior to test. Before 1 day, testing rats were briefly anesthetized with halothane, and a microdialysis probe was inserted into the guide cannula. Microdialysis probes were perfused overnight with artificial CSF (Song et al, 1999). The collection of dialysates began in 18-24 h later. In total, 12 samples were collected in two consequent conditions, baseline (5 samples) and injection of saline or IL-1 (7 samples). The interval between each sample was $30 \mathrm{~min}$. Following the collection, samples were immediately injected into a HPLC with electrochemical detector. The concentration of DA, 3,4-dihydroxyphenylacetic acid (DOPAC) and homovanillic acid (HVA) were determined in the dialysates according to a procedure previously described by Song et al (1999).

In experiment 2, 18 rats were divided into two groups of nine rats with the control diet. The surgery procedure was the same as the Exp 1. RU 486 was injected (s.c.) $10 \mathrm{~min}$ before IL-1 administration (Breivik et al, 2000; Song et al, 2004). The dialysates collection and measuring procedure were the same as the experiment 1.

In experiment 3, 56 animals were divided into four groups of 14 rats (in each group, the hypothalamus from seven rats were used for the measurement of PLA2 activities, and the other seven samples were used for PGE2 assay). The diets and the surgery were the same as the experiment 1 . After feeding for 49 days, rats were killed and brains were removed $1.5 \mathrm{~h}$ following systemic saline or IL-1 administration (because at this time point the concentration of

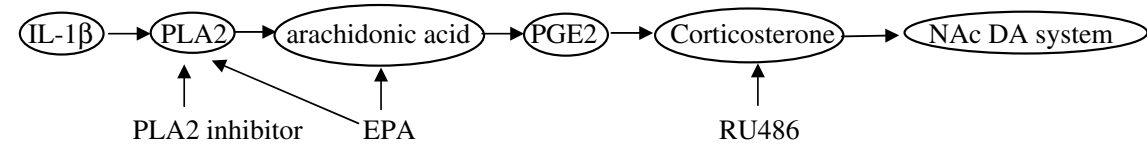

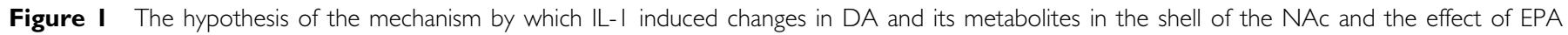
treatment. 
neurotransmitters and metabolites was significantly changed, and IL-1-induced mRNA expressions related to PGE2, glucocorticoids and inflammation in the brain also occurred) (Song et al, 1999; Song and Horrobin, 2004; Blais et al, 2002; Zhang and Rivest, 2000). Following decapitation, blood was collected and serum samples were used to measure corticosterone concentrations.

In experiment 4, 16 animals were divided into two groups of eight rats with control diet. One group was injected with saline and the other group with quinacrine $(30 \mathrm{mg} / \mathrm{kg})$, a nonselective PLA2 inhibitor, i.p. for 4 days, and the last injection was $30 \mathrm{~min}$ before IL-1 administration (Tariq et al, 2001). PGE2 and corticosterone concentration were measured $1.5 \mathrm{~h}$ after IL-1 injection.

\section{Animals and Diets}

Male Sprague-Dawley rats (200-220 g and 2 months old at the start of the experiment) were purchased from Charles River, Quebec, Canada. Food and water were available ad libitum. The colony was maintained at $21 \pm 1^{\circ} \mathrm{C}$ with a $12 \mathrm{~h}$ light-dark cycle (07:30-19:30 h). Animals were handled daily. The research protocol was approved by the Animal Care Committees of the University of British Columbia and the University of Prince Edward Island, Canada, and conformed to the guidelines of the Canadian Council for Animal Care.

Palm oil (1\%) that contained negligible amount of $n-3$ and n- 6 fatty acids was added to the rat chow as a control diet to ensure comparable texture and caloric value as $1 \%$ EPA diet. Palm oil (Harlan Teklad Test Diet, USA) and ethyl-EPA (Amarin Neuroscience/Laxdale Ltd, UK) were stored in a $4^{\circ} \mathrm{C}$ refrigerator. Palm oil was melted in a water bath $\left(<50^{\circ} \mathrm{C}\right)$. The powder of rat chow was then mixed with the palm oil or EPA $(1 \% \mathrm{v} / \mathrm{w})$. The food was prepared every 3-4 days and stored at $4{ }^{\circ} \mathrm{C}(12,31)$. Feeding period was 49 days (Song et al, 2003). The rats were about 3.6 months old before experimental testing.

\section{Surgery}

Rats were anesthetized with ketamine $(100 \mathrm{mg} / \mathrm{kg}$, i.p. $)$ and xylazine $(20 \mathrm{mg} / \mathrm{kg}$, i.p.). A 20 -gauge guide cannula containing a removable 24-gauge obturator was stereotaxically implanted aimed at the shell of the NAc $(A P=1.7 \mathrm{~mm}$, $\mathrm{DV}=-7.8 \mathrm{~mm}, \mathrm{~L}=0.7 \mathrm{~mm}$ ) according to the Atlas of Paxinos and Watson (1986) and was secured to the skull with four screws and dental cement.

\section{Reagents}

Rat recombinant IL- $1 \beta$ was obtained from NIBSC, Potters Bar, UK (biological activity: $317 \mathrm{IU} / \mathrm{mg}$ ), and dissolved in sterile, pyrogen-free saline at doses of $1 \mu \mathrm{g} / 0.5 \mathrm{ml} / \mathrm{rat}$ and prepared for i.p. administration. This dose was based on previous studies in which we assessed the behavioral and monoamine release induced by IL-1 (Merali et al, 1997; Song et al, 1999). RU486 (Sigma, Canada) was dissolved with $99 \%$ ethanol and then diluted with sterile saline to $25 \mathrm{mg} / \mathrm{kg} / \mathrm{ml}$ (McCullers et al, 2002; Heikinheimo and Kekkonen, 1993). The final concentration of ethanol was $<1 \%$. Quinacrine (Sigma, Canada) was dissolved in saline as $30 \mathrm{mg} / \mathrm{kg}$ for i.p. injection (Tariq et al, 2001). Rats were gently handled daily for 1 week before the start of the i.p. or s.c. injection.

\section{PGE2 Assay}

Following weighing and sonicating in an ice-cold PBS, the hypothalamus samples were centrifuged at $7000 \mathrm{~g}$ at $4{ }^{\circ} \mathrm{C}$ for $30 \mathrm{~min}$. The supernatants were collected and frozen in a $-80^{\circ} \mathrm{C}$ freezer for the further PEG2 assays.

The concentration of PGE2 was measured by an enzyme immunoassay (EIA) (Assay Designs Inc., Ann Arbor, USA). The assay method was the same as the method described previously (Song et al, 1998). In brief, standards and samples $(50 \mu \mathrm{l})$ were pipetted into the wells of plates that coated with specific PGE2 antibodies and then incubated at $37^{\circ} \mathrm{C}$. After washing away any unbound ligand, an enzymelinked polyclonal antibody specific for PGE2 was added to the wells and incubated at $37^{\circ} \mathrm{C}$. Following removal of any unbound antibody-enzyme reagent, a substrate solution was added to the wells. The density of the color was proportional to the amount of protein bound. The intensity of the color was measured at $405 \mathrm{~nm}$ wavelength by a microtiter plate reader (Song et al, 1998).

\section{Measurement of cPLA2 and sPLA2 Activities}

The hypothalamus was homogenized with a sonicator in ice-cold PBS. The protein concentration was determined in the homogenates with a Coomassie Plus Protein Assay Kit (Pierce) according to the manufacturer's protocol.

The $\mathrm{cPLA}_{2}$ activity was measured with an assay kit (Cayman Chemicals), as described by Bosetti and Weerasinghe (2003). Briefly, $1.5 \mathrm{mM}$ arachidonoyl thio-PC (1-Ohexadecyl-2-deoxy-2-thio-R-(arachidonoyl)-sn-glyceral-3-phosphorylcholine) in the kit was used as a synthetic substrate to detect PLA2 activity. Each sample was homogenized in $0.5 \mathrm{ml}$ of ice-cold $10 \mathrm{mM}$ Tris buffer $\mathrm{PH} 7.8$ (1\% Nonidet P-40, 0.15 M NaCl, and $1 \mathrm{mM}$ EDTA, $2 \mathrm{mM}$ dithiothreitol, $10 \mu \mathrm{M}$ phenylmethylsulphonyl fluoride, $9.2 \mu \mathrm{M}$ aprotinin, $0.11 \mu \mathrm{M}$ leupeptin, and $10.2 \mu \mathrm{M}$ pepstatin A). Cytosolic fractions were obtained by centrifuging at $18000 \mathrm{~g}$ for $15 \mathrm{~min}$ at $4^{\circ} \mathrm{C}$ (Rintala et al, 1999). cPLA 2 activity was determined in the cytosolic fractions with the presence of the $\mathrm{iPLA}_{2}$-specific inhibitor bromoenol lactone (BEL, $\left.10 \mu \mathrm{M}\right)$ and the $s \mathrm{PLA}_{2}$-specific inhibitor thioetheramide-PC (TEPC, $50 \mu \mathrm{M}$ ), which were incubated with the samples for $20 \mathrm{~min}$ at $25^{\circ} \mathrm{C}$ prior to the assay. According to Ghelardoni and coinvestigators' method, to confirm the reliability of this assay for cPLA2, the assay was repeated and verified in the presence of $50 \mu \mathrm{mol} / \mathrm{l}$ cPLA2 specific inhibitor arachidonyl trifluoromethyl ketone (AACOCF3) (Ghelardoni et al, 2004). sPLA 2 activity was measured with an $\mathrm{sPLA}_{2}$ assay kit (Cayman Chemicals) (Bosetti and Weerasinghe (2003). 1,2-diheptanoyl thio-PC (1,2-bis (heptanoyl)-1,2dideoxy-sn-glyceral-3-phosphorylcholine) in the kit was used to serves as a substrate for $\mathrm{SPLA}_{2}$ enzymes but not for $\mathrm{CPLA}_{2}$. Bee venom $\mathrm{PLA}_{2}$ was run as a positive control in the same assay to show a linear increase in the absorbance over the time range chosen. In both cPLA2 and sPLA2 measurements, Ellman's reagent was used to detect free thiol that was released by the hydrolysis of the diheptanoyl 
thioesther bond at sn-2 position. Absorbance was measured every minute after adding the substrate. Activity was calculated by measuring the absorbance at $414 \mathrm{~nm}$, using the DTNB extinction coefficient of 10.66 per $\mathrm{mM} / \mathrm{l} / \mathrm{cm}$, and units are expressed as $\mathrm{nmol} / \mathrm{min} / \mathrm{g}$ of cytosolic protein. Each sample was measured in duplicate. Results are expressed as percentage of the control.

\section{Measurement of Serum Corticosterone Concentration}

Serum samples from trunk blood of experiment 3 were used for the corticosterone measurement with a commercial radioimmunoassay kit (Immuchem corticosterone RIA kit for rats; catalogue No. RCBK9906A; ICN Biochemical, Costa Mesa, CA, USA). Intra- and interassay coeffients of variation were 6.8 and $5.6 \%$, respectively.

\section{Verification of Probe Placement}

Upon completion of the experiments 1 and 2, rats were anesthetized with an overdose of phenol-barbitone, and perfused intracardially with saline followed by $10 \%$ formalin. Brains were removed and sliced in $30 \mathrm{~mm}$ coronal section. Brain slides were stained with Thionine for verification of probe placement. Only experimental data from rats whose probe tips were confirmed within the shell of the NAc were considered for analysis. About $10 \%$ of animals were discarded.

\section{Data Analysis}

The body weight was measured by one-way repeated ANOVA. In the experiments 1 and 2, the baseline dialysate samples were averaged as baseline and subsequent data points for each rat were expressed as a percentage of this baseline score. The data including the baseline samples, were then analyzed by three-way repeated ANOVA for experiments 1 and 2 (treatment $\times$ sampling times $\times$ diet). In experiments 3 and 4 , results were analyzed by two-way ANOVA (treatment $\times$ IL-1). A Newman-Keuls post hoc was conducted for multiple comparisons between the groups.

\section{RESULTS}

\section{Gain of Body Weight}

At 3 days after surgery, the gain of body weight was significantly reduced in the group of rats fed palm oil $(-3$. $4 \pm 0.31 \mathrm{~g}$ ) when compared to the EPA feeding animals $(2.65 \pm 0.23 \mathrm{~g})(\mathrm{F} 1,35=12.43 ; p<0.01)$. Single peripheral IL1 administration did not significantly reduce animal body weight (result not shown). Animals showed shivering behavior in the testing cage for several hours after IL-1 administration.

\section{Basal Levels of Dopamine and Metabolites in the NAc}

There was no significant difference in the interstitial concentrations of DA, DOPAC and HVA between two groups with different diets (rat chow plus $1 \%$ palm oil and rat chow plus 1\% EPA) (Table 1). Data were expressed as the absolute concentration in the last baseline samples.
Table I Basal Concentrations of DA and its Metabolite in the Shell of the NAc after Feeding Control and I\% EPA Diets for 49 Days

\begin{tabular}{lccc}
\hline Groups & DA $(\mathbf{p g} / \mathbf{4 0} \boldsymbol{\mu l})$ & DOPAC $(\mathbf{n g} / \mathbf{4 0} \boldsymbol{\mu l})$ & HVA $(\mathbf{n g} / \mathbf{4 0} \boldsymbol{\mu l})$ \\
\hline Control diet & $6.43 \pm 1.74$ & $7.25 \pm 1.61$ & $7.82 \pm 1.37$ \\
EPA diet & $4.56 \pm 0.75$ & $5.88 \pm 0.72$ & $5.62 \pm 0.84$ \\
\hline
\end{tabular}

Results are expressed as mean $\pm \operatorname{SEM}(n=9)$.

\section{EPA Significantly Attenuated IL-1 $\beta$-Induced Increase in} Extracellular DA Concentration

ANOVA analysis indicated that there was a significant interaction between injection and sampling time (F9, $108=7.34, p<0.01)$. Newman-Keuls post hoc showed that saline injection result in a small but significant increase in DA release $(p<0.05)$, while systemic IL-1 administration induced a more pronounced increase in DA release $(p<0.05 ; 0.01)$ (Figure $2 \mathrm{a})$. The ANOVA analysis indicated that the diet factor did not exert any significant effect on DA release. However, the interaction between injection and diet was significant $(\mathrm{F} 9,108=4.78, p<0.05)$. Multiple comparisons with Newman-Keuls showed that EPA significantly attenuated the extracellular concentration of DA in both saline and IL-1-treated groups $(p<0.05$ or 0.01$)$ (Figure $2 b)$.

\section{EPA Significantly Attenuated IL-1 $\beta$ Induced Increase in DOPAC Level}

The ANOVA analysis indicated that there was a significant interaction between injection and sampling time in extracellular level of DOPAC (F9, $108=6.93, p<0.01)$. NewmanKeuls post hoc analysis between groups revealed that a small but significant increase $(120 \%)$ in extracellular concentration of DOPAC occurred after saline injection $(p<0.05)$ as compared to average percent of baseline values $(100 \%)$, while IL-1-increased the DOPAC concentration was greater $(133 \%)$ than saline injection $(120 \%)(p<0.01)$ (result's not shown). The ANOVA analysis also revealed a significant EPA and IL-1 interaction (F9, $108=6.32, p<0.01)$. Multiple comparisons between groups indicated that IL-1-induced increase in the DOPAC level was significantly attenuated in EPA feeding animals $(105 \%, p<0.05)$ (result not shown).

\section{EPA Significantly Attenuated IL-1 Induced Increase in HVA}

The ANOVA analysis indicated a significant interaction between IL-1 or saline injection and sampling time in extracellular HVA levels (F9, $108=23.91, p<0.0001)$. The multiple comparison showed a small but significant increase in HVA levels (117\%) $2 \mathrm{~h}$ following saline injection when compared to the percentage of baseline values $(p<0.05)$ (result not shown). At $1 \mathrm{~h}$ following IL-1 $\beta$ administration, significant rise of HVA levels was observed $(127 \%)(p<0.01)$. This increase was more pronounced and longer lasting than saline treatment $(p<0.05$ at injecting points 3 and 4 ). The ANOVA analysis showed that no significant change occurred in the interaction between IL-1/ saline administration and sampling time in EPA fed group 


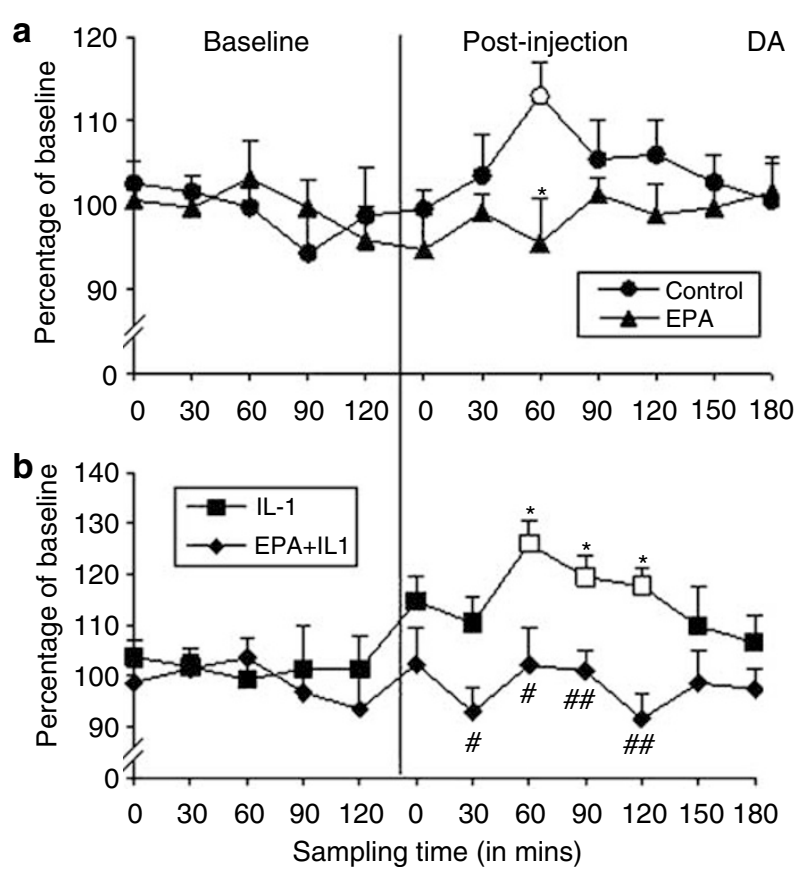

Figure 2 Saline or IL-I induced changes in DA release from NAc shell of rats fed palm oil ( $1 \%$ of diet) or EPA ( $1 \%$ of diet) for 49 days. Results are expressed as percent changes from baseline (initial five samples). After collection of five baseline samples, rats were injected with saline $(0.5 \mathrm{ml} / \mathrm{rat})$ or $\mathrm{IL}-\mathrm{I} \beta(\mid \mu \mathrm{g} / 0.5 \mathrm{ml} / \mathrm{rat})$, and seven samples were collected. (a) The comparison between palm oil and EPA with saline treatment; circles represent group fed with palm oil diet; triangles represent group fed with EPA. (b) The comparison between palm oil and EPA diet groups with IL-I injection. Squares denote animals with palm oil; diamonds denote with EPA diet. Opened forms represent significant differences $(p<0.05$ or $0.0 \mathrm{l}$ ) from the average of baseline samples (solid forms). $* p<0.05$ vs saline-treated rats fed palm oil (in a) $(n=8-9) ;{ }^{\#} p<0.05,{ }^{\# \#} p<0.0$ I vs IL-I-treated group fed with palm oil $(n=8-9)$.

but revealed that the interaction between EPA, IL-1 and sampling time was significant $(\mathrm{F} 9,108=6.51, p<0.01)$. In the group fed EPA diet, the rise of HVA level was significantly attenuated after saline $(97 \%)(p<0.05)$ or IL-1 $\beta$ administration $(106 \%)(p<0.01)$ when compared to these groups fed palm oil (result not shown).

\section{RU486 Significantly Attenuated IL-1 $\beta$ Induced Increase in DA and its Metabolite}

In this experiment, the pattern of DA, DOPAC and HVA in response to IL-1 administration was similar to the results described in experiment 1 . Systemic IL-1 administration significantly increased extracellular concentrations of DA and its metabolites (DA: F10,96 $=7.73, p<0.01$; DOPAC: $\mathrm{F} 10,96=6.94, p<0.01$; HVA: $\mathrm{F} 10,96=16.83, p<0.001)$. The ANOVA analysis also showed a significant interaction between IL-1 and RU486 in extracellular DA, DOPAC and HVA concentrations (DA: F10, $96=8.62, p<0.01$; DOPAC: F10, $96=9.35, p<0.01$; HVA: F10, $96=11.49, p<0.001)$. Newman-Keuls post hoc comparison indicated a significant difference in DA, DOPAC and HVA between two groups $1-2 \mathrm{~h}$ following IL-1 administration. At $1 \frac{1}{2} \mathrm{~h}$ following IL-1 administration, the DA concentration was increased $(p<0.05)$, which was reduced in group pretreated with RU486 $(p<0.05)$ (Figure 3). The DOPAC concentration was

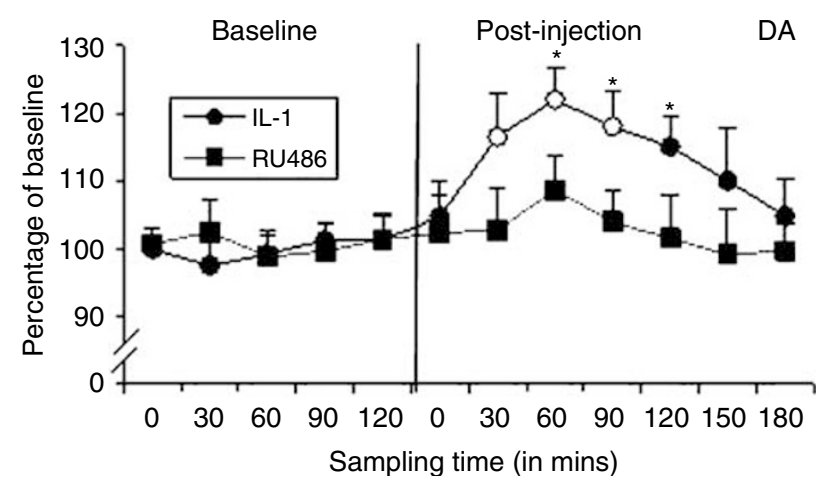

Figure 3 Effects of RU486 ( $25 \mathrm{mg} / \mathrm{kg}$, s.c.) on extracellular concentrations of DA in the shell after $|\mathrm{L}-|$ administration ( $\mid \mu g / 0.5 \mathrm{ml} / \mathrm{rat}$, i.p.). Circles represent group treated with saline and IL-I; Squares denote group treated with RU486 and IL-I. Opened forms represent significant differences $(p<0.05$ or 0.01$)$ from the average of baseline samples (solid forms). ${ }^{*} p<0.05$ vs the group treated with RU486 $(n=8)$.

increased to $127.7 \%$, whereas reduced to $106 \%$ in the group treated with RU486 (result not shown). IL-1 administration also induced an elevation in extracellular level of HVA (128.6\%), which was attenuated to $109 \%$ after RU486 treatment (result not shown).

\section{Effects of EPA on Activities of cPLA2 and SPLA2 after IL-1 Administration}

Two-way ANOVA indicated that a significant interaction between IL-1 and these enzymes (cPLA2: F1,27 $=32.21$, $p<0.0001$; sPLA2: F1,27 $=25.06, p<0.001$ ). Newman-Keuls comparison between groups showed that systemic IL-1 administration significantly increased cPLA2 and sPLA2 activities $(p<0.001)$ (Figure 4$)$. EPA alone did not exert significant effect on these enzyme activities. ANOVA analysis also revealed a significant interaction between IL1 and EPA (cPLA2: F1,27 $=22.92, p<0.001$; sPLA2: F1,27 $=$ $14.33 ; p<0.01)$. Newman-Keuls post hoc showed EPA significantly reduced PLA2 activities in IL-1-treated group $(p<0.01)$ (Figure 4).

\section{The Modulation of EPA on IL-1 Induced Changes in the PGE2 Concentration}

Different diets had no significant effect on hypothalamic PGE2 concentrations in saline-treated animals. Comparing to control group (saline and palm oil), IL-1 $\beta$ administration significantly increased accumbal PGE2 $(F 2,30=8.96$, $p<0.01)$ and hypothalamic PGE2 (F2,30 $=9.84, p<0.01)$ (Table 2). In EPA feeding group, IL-1 induced change in PGE2 was significantly attenuated (F2,30 $=8.51, p<0.01)$ (Table 2).

\section{EPA Attenuated IL-1 Induced Increase in Serum Corticosterone Concentrations}

Table 3 shows that systemic administration of IL- $1 \beta$ at dose $1 \mu \mathrm{g}$ markedly increased serum corticosterone concentrations when compared to saline-treated animals (F2, 34= 9.62, $p<0.01)$. The ANOVA analysis also revealed that EPA treatment significantly attenuated IL-1 induced rise of corticosterone $(\mathrm{F} 2,34=7.34, p<0.01)$ (Table 3$)$. 

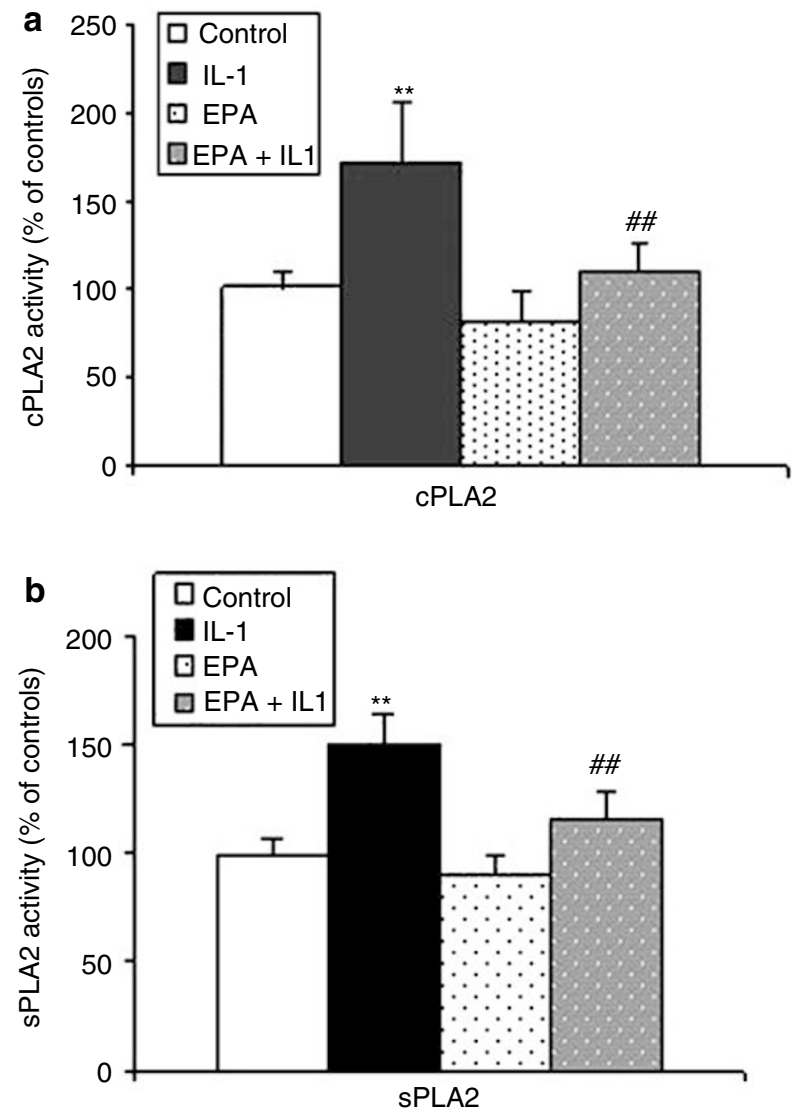

Figure 4 The effect of EPA ( $1 \%$ of diet) fed for 49 days on the activity of (a) CPLA2 and (b) sPLA2 enzymes in the hypothalamus after saline or IL-I administration $(1 \mu \mathrm{g} / 0.5 \mathrm{ml} / \mathrm{rat}$ ). $* * * 0.01$ vs the group fed palm oil and injected with saline $(n=7)$; ${ }^{\# \#} p<0.01$ vs group fed palm oil and injected with IL-I $(n=7)$.

Table 2 Effects of I\% EPA Fed for 49 Days or Quinacrine $(30$ mg/ kg) Treatment for 4 Days on Hypothalamic PGE2 Concentration

\section{Groups}

Palm oil+Saline

Palm oil+IL-I $\beta$

EPA+saline

EPA+IL-I $\beta$

IL-I+saline

IL-I +quinacrine
Hypothalamic PGE2 (ng/g brain tissue)

$$
\begin{gathered}
4.67 \pm 0.36 \\
9.76 \pm 1.05^{*} \\
3.54 \pm 0.31 \\
5.25 \pm 0.49^{\#} \\
10.84 \pm 1.22 \\
5.72 \pm 0.56^{\#}
\end{gathered}
$$

Results are expressed as mean $\pm \mathrm{SEM}$.

${ }^{*} p<0.05$ vs control (saline); ${ }^{\#}<0.05$ vs IL-I group $(n=8-9)$.

\section{Quinacrine Significantly Attenuates IL-1 Effects on PGE2 and Corticosterone Concentrations}

ANOVA analysis showed a similar increase in hypothalamic PGE2 $(\mathrm{F} 1,15=15.62, p<0.01)$ and corticosterone levels $(\mathrm{F} 1,15=14.82, p<0.01)$ after IL-1 administration in this experiment. Quinacrine pretreatment significantly attenuated these increases (PGE2: $\mathrm{F} 1,15=13.74, \quad p<0.01$; corticosterone: $\mathrm{F} 1,15=12.77, p<0.01$ ) (Tables 2 and 3 , respectively).

\section{DISCUSSION}

The present study, for the first time, has demonstrated that (i) the response of shell dopaminergic system to IL-1 $\beta$ challenge is similar to its response to a stressor ( $\mathrm{Wu}$ et al, 1999; Barrot et al, 2000); (ii) the shell is more responsive to IL-1 stimulation than the core since previously we have reported that IL-1 has no effect on DA and DOPAC in the core (Song et al, 1999); (iii) the effect of IL-1 on DA and metabolites may result from the activation of PLA2-PGE2corticosterone pathway because the PLA inhibitor blocked IL-1 effects on PGE2 and corticosterone, while the glucocorticoid receptor antagonist blocked IL-1 effect on the interstitial concentrations of DA and metabolites and (iv) EPA attenuated IL-1 induced changes by inhibiting the interaction of PLA2-PGE2-corticosterone.

Our previous studies have reported that IL- 1 can induce anxiety or stress-like behaviors and impair memory (Song et al, 2003, 2004). Anatomic and pharmacological studies have shown that the shell is interconnected with the limbic system (Nirenberg et al, 1997; Jackson and Moghaddam, 2001), which is involved in mood disorder and cognitive impairment (Schwartz et al, 2003). A glucocorticoid/ dopamine hypothesis for depression was raised in 1980's (Schatzberg et al, 1985). Therefore, one of the major findings of this study is to demonstrate a relationship between PLA2-PGE2-corticosterone and shell DA and metabolites, which may play an important role in inflammation-induced mental disorders.

Previous studies have reported that stress can increase extracellular DA and DOPAC concentrations in the NAc shell (Kalivas and Duffy, 1995; Wu et al, 1999). Similar results have been also found in the present study after saline injection (as a mild stress). Similar but greater responses of DA and its metabolites have been found following IL-1 administration. Increased DA and its metabolites may indicate the activation of DA neurons such as an increase in DA synthesis and metabolism, and decrease in DA reuptake. DA response to a stressor only in the shell but not in the core

\begin{tabular}{|c|c|c|c|c|c|}
\hline Palm oil+saline & Palm oil+IL-I & EPA+saline & EPA+IL-I & IL-I+ saline & IL-I+Quinacrine \\
\hline
\end{tabular}
has been explained as the stimulation of glucocorticoids. Barrot et al (2000) reported that saline or drug injection (as a mild stress) increased DA release could be prevented

Table 3 Effects of I\% EPA Diet Fed for 49 Days or Quinacrine $(30 \mathrm{mg} / \mathrm{kg})$ for 4 Days on the Corticosterone Concentration after IL-I $\beta$ Administration

Results are expressed as mean \pm SEM $(\mu \mathrm{g} / \mathrm{d})$ ).

${ }^{*} p<0.0$ I vs coconut oil \pm saline; ${ }^{\#} p<0.0$ I vs coconut oil \pm IL-I $(n=8-9)$. 
in adrenalectomised rats or be restored after corticosterone administration. In addition, Rouge-Pont et al (1999) reported that RU 39305, a glucocorticoid receptor antagonist, could suppress DA release from mesencephalic dopaminergic neurons. As mentioned in the introduction, IL-1 stimulates glucocorticoid secretion through PGE2 and CRF (Engblom et al, 2002). Increases in corticosterone and PGE2 in the hypothalamus have been observed in the present study. Our results from the RU486 experiment further support the relationship between shell DA system and corticosterone, and also demonstrate that an increase in the release of DA and metabolites is related to IL-1 effect on the corticosterone secretion. In the present study, we did not measure absolute concentrations of DA and metabolites in the group treated with RU486 because our previous and other's studies did not find that acute RU486 could lead to any behavioral change (Marinelli et al, 1998; Song et al, 2004). Different from our and other's findings (Marinelli et al, 1998), Imperato et al (1991) did not find that corticosterone play a role in DA release from the NAc since RU 486 did not attenuate stress-induced changes. However, since that paper did not provide which part of NAc was studied and what RU486 dose was used, it is difficult to compare our results with theirs.

It is known that three types of PLA2 enzymes are distributed in neurons and glia cells in many brain regions including the hypothalamus, and are involved in neuroinflammation. The functions of CPLA2 and sPLA2 are $\mathrm{Ca}^{2+}$ dependent (Sun et al, 2004; Phillis and O'Regan, 2004). As our previous study has shown that IL-1 effects on glutamate receptor expressions in hippocampal neurons are $\mathrm{Ca}^{2+}$ dependent (Lai et al, 2006), and n-6 fatty acid AA can activate $\mathrm{Ca}^{2+}$ dependent PLA2 expressions in inflammation (Strokin et al, 2003), we chose to study these two enzymes. The major function of cPLA2 is to release AA, the precursor of eicosanoids that produces PGE2 and inflammatory cytokines (Xu et al, 2003). AA can also modulate neurotransmitter release, activate the HPA axis and change behavior (Smith et al, 1994; Narumiya et al, 1999). sPLA2 has been shown to participate in the PGE2 production (Kuwata et al, 1998) and involved in cerebral ischemia and neuroinflammation (Lin et al, 2004; Rosenberger et al, 2004). LPS and inflammatory cytokines can trigger the activity and expression of these PLA2 enzymes (Morioka et al, 2002; Rosenberger et al, 2004). We have also previously reported that central IL-1 administration significantly increased PGE2 concentration in the brain (Song and Horrobin, 2004). Therefore, it is not surprising that systemic administration of IL-1 significantly increased both PLA2 enzyme activities and PGE2 synthesis in the hypothalamus in the present study. The increase in hypothalamic PGE2 further supports that IL-1-induced secretion of corticosterone through PGE2 synthesis in the brain. The mechanism by which IL-1 influences brain functions have been reported as (i) passing the blood-brain barrier to combine its receptors that are widely contributed in many brain regions (Saija et al, 1995; Blatteis, 2000; Pan and Kastin, 2003) and (ii) activating the vagus nerves (Hansen et al, 1998; Luheshi et al, 2000).

The present study also for the first time demonstrated that EPA enriched diet significantly attenuate changes in shell DA and metabolites following IL-1 administration. One mechanism may be the inhibiting effect of EPA on the interaction of PLA2-PGE2-corticosterone, because results from present study have demonstrated that EPA significantly reduced PLA2 activities and PGE2 levels in the hypothalamus, and decreased serum corticosterone concentration, which were increased by IL-1 administration. These results are consistent with our previous findings that EPA reduces PGE2 synthesis in the hippocampus and hypothalamus, and block corticosterone secretion after central IL-1 administration (Song et al, 2003, 2004). So far, direct effects of EPA on PLA2 expressions or activities have not been studied in the brain. A clinical study has reported that increased blood concentrations of n-6 fatty acids and sPLA2 in children with autistic spectrum disorders can be reduced by EPA treatment (Bell et al, 2004). In peritoneal macrophages, EPA has been shown to inhibit PLA2-related signal expressions (Tappia et al, 1995). The other mechanism by which EPA attenuated IL-1 effects may be via its competition of n-6 fatty acid AA that may have direct effect on the PLA2 expression, PGE2, HPA axis and neurotransmission (Yokotani et al, 2000). Over-taken of n-6 fatty acids has been associated with higher risk of autoimmune and psychiatric diseases (James et al, 2000; Calder and Grimble, 2002). The evidence from a human study has shown subjects with lower serum n-3 fatty acid levels or with a higher $n-6 / n-3$ ratio had a significantly higher stressinduced increase in some proinflammatory cytokines (Maes et $a l, 2000)$. Decreased n-3 fatty acids and increased n-6/n-3 ratio have been reported in patients with depression and Alzheimer's disease (Maes et al, 1999; Tully et al, 2003). Owing to condition limitation, we could not measure the AA concentration in the shell after EPA treatment. However, several studies have demonstrated that EPA treatment can decrease AA concentration or $n-6 / n-3$ ratio in different regions of the brain (Naliwaiko et al, 2004; Amusquivar et al, 2000).

In the group treated with saline, EPA also significantly attenuated changes in DA and its metabolites induced by saline injection. We have previously reported that saline injection acted as a mild stressor (Song et al, 1999). In the present study, saline-induced changes were less but similar to IL-1 induced changes. Many previous studies have shown that IL-1 induced changes in neurotransmission are similar to those after stress exposure (Song et al, 1999; Anisman et al, 2002; Dunn, 2000). It is known that stress not only triggers glucocorticoid secretion but also increases inflammatory cytokine synthesis (Grippo et al, 2005; O'Connor et al, 2003). As demonstrated by this study, EPA may modulate inflammation and corticosterone pathway, similar mechanism may be also involved in the EPA reduced DA and its metabolites after saline injection.

In summary, the present study demonstrated that systemic IL-1 administration increased extracellular DA, DOPAC and HVA concentrations in the NAc shell. These changes may result from IL-1 stimulation of corticosterone secretion since RU486 block IL-1 effect. cPLA2 and sPLA2 enzymes are involved in this procedure since quinacrine blocked IL-1 induced increases in PGE2 and corticosterone. Ethyl-EPA treatment significantly attenuated IL-1 induced changes by the inhibition of PLA-PGE2-corticosterone pathway. These results further support the idea that polyunsaturated fatty acids could be developed as a new therapy for some psychiatric and inflammation associated disorders. 


\section{ACKNOWLEDGEMENTS}

The authors have spent 4 years to finish this study and received great attention and support from Dr David Horrobin. The authors wish to publish this paper in memory of Dr David Horrobin who past away three years ago. We thank Professor AG Phillips for providing facilities to enable a part of this study to be undertaken, and thank Dr Pornnarin Taepavarapruk for her kind help with the analysis of some microdialysis samples. This project was supported by a grant from Amarin Neuroscience/ Laxdale Ltd, Scotland, UK and Canadian Institutes for Health Research, Canada.

\section{REFERENCES}

Altman J, Everitt BJ, Glautier S, Markou A, Nutt D, Oretti R et al (1996). The biological, social and clinical bases of drug addiction: commentary and debate. Psychopharmacology 125: 285-345.

Amusquivar E, Ruperez FJ, Barbas C, Herrera E (2000). Low arachidonic acid rather than alpha-tocopherol is responsible for the delayed postnatal development in offspring of rats fed fish oil instead of olive oil during pregnancy and lactation. J Nutr 130: 2855-2865.

Anisman H, Kokkinidis L, Merali Z (2002). Further evidence for the depressive effects of cytokines: anhedonia and neurochemical changes. Brain Behav Immun 16: 544-556.

Barrot M, Marinelli M, Abrous DN, Rouge-Pont F, Le Moal M, Piazza PV (2000). The dopaminergic hyper-responsiveness of the shell of the nucleus accumbens is hormone-dependent. Eur J Neurosci 12: 973-979.

Bell JG, MacKinlay EE, Dick JR, MacDonald DJ, Boyle RM, Glen AC (2004). Essential fatty acids and phospholipase A2 in autistic spectrum disorders. Prostaglandins Leukot Essent Fatty Acids 71: 201-204.

Brebner K, Hayley S, Zacharko R, Merali Z, Anisman H (2000). Synergistic effects of interleukin-1beta, interleukin-6, and tumor necrosis factor-alpha: central monoamine, corticosterone, and behavioral variations. Neuropsychopharmacology 22: 566-580.

Breivik T, Thrane PS, Gjermo P, Opstad PK (2000). Glucocorticoid receptor antagonist RU 486 treatment reduces periodontitis in Fischer 344 rats. J Periodontal Res 35: 285-290.

Bland ST, Twining C, Watkins LR, Maier SF (2003). Stressor controllability modulates stress-induced serotonin but not dopamine efflux in the nucleus accumbens shell. Synapse 49: 206-208.

Blais V, Zhang J, Rivest S (2002). In altering the release of glucocorticoids, ketorolac exacerbates the effects of systemic immune stimuli on expression of proinflammatory genes in the brain. Endocrinology 143: 4820-4827.

Blatteis CM (2000). The afferent signalling of fever. J Physiol 526: 470 .

Bosetti F, Weerasinghe GR (2003). The expression of brain cyclooxygenase-2 is down-regulated in the cytosolic phospholipase A2 knockout mouse. J Neurochem 87: 1471-1477.

Calder PC, Grimble RF (2002). Polyunsaturated fatty acids, inflammation and immunity. Eur J Clin Nutr 56: S14-S19.

Dantzer R, Bluthe RM, Gheusi G, Cremona S, Laye S, Parnet P et al (1998). Molecular basis of sickness behavior. Ann NY Acad Sci 856: $132-138$.

Delarue J, Matzinger O, Binnert C, Schneiter P, Chiolero R, Tappy L (2003). Fish oil prevents the adrenal activation elicited by mental stress in healthy men. Diabet Metab 29: 289-295.

Diez E, Louis-Flamberg P, Hall RH, Mayer RJ (1992). Substrate specificities and properties of human phospholipases A2 in a mixed vesicle model. J Biol Chem 267: 18342-18348.
Dunn AJ (2000). Cytokine activation of the HPA axis. Ann NY Acad Sci 917: 608-617.

Egan MF, Weinberger DR (1997). Neurobiology of schizophrenia. Curr Opin Neurobiol 7: 701-707.

Engblom D, Ek M, Saha S, Ericsson-Dahlstrand A, Jakobsson PJ, Blomqvist A (2002). Prostaglandins as inflammatory messengers across the blood-brain barrier. J Mol Med 80: 5-15.

Fulford AJ, Marsden CA (1998a). Conditioned release of 5 -hydroxytryptamine in vivo in the nucleus accumbens following isolation-rearing in the rat. Neuroscience 83: 481-487.

Fulford AJ, Marsden CA (1998b). Effect of isolation-rearing on conditioned dopamine release in vivo in the nucleus accumbens of the rat. J Neurochem 70: 384-390.

Ghelardoni S, Tomita YA, Bell JM, Rapoport SI, Bosetti F (2004). Chronic carbamazepine selectively downregulates cytosolic phospholipase A2 expression and cyclooxygenase activity in rat brain. Biol Psychiatry 56: 248-254.

Green P, Gispan-Herman I, Yadid G (2005). Increased arachidonic acid concentration in the brain of Flinders Sensitive Line rats, an animal model of depression. J Lipid Res 46: 1093-1096.

Grippo AJ, Francis J, Beltz TG, Felder RB, Johnson AK (2005). Neuroendocrine and cytokine profile of chronic mild stressinduced anhedonia. Physiol Behav 84: 697-706.

Hansen MK, Taishi P, Chen Z, Krueger JM (1998). Vagotomy blocks the induction of interleukin-1beta (IL-1beta) mRNA in the brain of rats in response to systemic IL-1beta. J Neurosci 18: 2247-2253.

Heikinheimo O, Kekkonen R (1993). Dose-response relationships of RU 486. Ann Med 25: 71-76.

Heimer L, Alheid GF, de Olmos JS, Groenewegen HJ, Haber SN, Harlan RE et al (1997). The accumbens: beyond the core-shell dichotomy. J Neuropsychiatr Clin Neurosci 9: 354-381.

Jackson ME, Moghaddam B (2001). Amygdala regulation of nucleus accumbens dopamine output is governed by the prefrontal cortex. J Neurosci 21: 676-681.

James MJ, Gibson RA, Cleland LG (2000). Dietary polyunsaturated fatty acids and inflammatory mediator production. Am J Clin Nutr 71: S343-S348.

Kalivas PW, Duffy P (1995). Selective activation of dopamine transmission in the shell of the nucleus accumbens by stress. Brain Res 675: 325-328.

Kuwata H, Nakatani Y, Murakami M, Kudo I (1998). Cytosolic phospholipase A2 is required for cytokine-induced expression of type IIA secretary phospholipase A2 that mediates optimal cyclooxygenase-2-dependent delayed prostaglandin E2 generation in rat 3 Y1 fibroblasts. J Biol Chem 273: 1733-1740.

Lai AY, Swayze RD, El-Husseini A, Song C (2006). Interleukin-1 beta modulates AMPA receptor expression and phosphorylation in hippocampal neurons. J Neuroimmun (in press).

Lin TN, Wang Q, Simonyi A, Chen JJ, Cheung WM, He YY et al (2004). Induction of secretary phospholipase A2 in reactive astrocytes in response to transient focal cerebral ischemia in the rat brain. J Neurochem 90: 637-645.

Luheshi GN, Bluthe RM, Rushforth D, Mulcahy N, Konsman JP, Goldbach $\mathrm{M}$ et al (2000). Vagotomy attenuates the behavioural but not the pyrogenic effects of interleukin-1 in rats. Auton Neurosci 85: 127-132.

Maes M, Christophe A, Bosmans E, Lin A, Neels H (2000). In humans, serum polyunsaturated fatty acid levels predict the response of proinflammatory cytokines to psychologic stress. Biol Psychiatry 47: 910-920.

Maes M, Christophe A, Delanghe J, Altamura C, Neels H, Meltzer HY (1999). Lowered omega3 polyunsaturated fatty acids in serum phospholipids and cholesteryl esters of depressed patients. Psychiatr Res 85: 275-291.

Marinelli M, Aouizerate B, Barrot M, Le Moal M, Piazza PV (1998). Dopamine-dependent responses to morphine depend on glucocorticoid receptors. Proc Natl Acad Sci USA 95: 7742-7747. 
McCullers DL, Sullivan PG, Scheff SW, Herman JP (2002). Mifepristone protects CA1 hippocampal neurons following traumatic brain injury in rat. Neuroscience 109: 219-230.

Merali Z, Lacosta S, Anisman H (1997). Effects of interleukin-1beta and mild stress on alterations of norepinephrine, dopamine and serotonin neurotransmission: a regional microdialysis study. Brain Res 761: 225-235.

Morioka N, Takeda K, Kumagai K, Hanada T, Ikoma K, Hide I et al (2002). Interleukin-1beta-induced substance $P$ release from rat cultured primary afferent neurons driven by two phospholipase A2 enzymes: secretory type IIA and cytosolic type IV. J Neurochem 80: 989-997.

Naliwaiko K, Araujo RL, da Fonseca RV, Castilho JC, Andreatini R, Bellissimo MI et al (2004). Effects of fish oil on the central nervous system: a new potential antidepressant? Nutr Neurosci 7: 91-99.

Narumiya S, Sugimoto Y, Ushikubi F (1999). Prostanoid receptors: structures, properties, and functions. Physiol Rev 79: $1193-1226$.

Nestler EJ, Barrot M, DiLeone RJ, Eisch AJ, Gold SJ, Monteggia LM (2002). Neurobiology of depression. Neuron 34: 13-25.

Nirenberg MJ, Chan J, Pohorille A, Vaughan RA, Uhl GR, Kuhar MJ et al (1997). The dopamine transporter: comparative ultrastructure of dopaminergic axons in limbic and motor compartments of the nucleus accumbens. J Neurosci 17: 6899-6907.

Noguchi T, Yoshida Y, Chiba S (2001). Effects of psychological stress on monoamine systems in subregions of the frontal cortex and nucleus accumbens of the rat. Brain Res 916: 91-100.

O'Connor KA, Johnson JD, Hansen MK, Wieseler Frank JL, Maksimova E, Watkins LR et al (2003). Peripheral and central proinflammatory cytokine response to a severe acute stressor. Brain Res 991: 123-132.

Pan W, Kastin AJ (2003). Interactions of cytokines with the blood-brain barrier: implications for feeding. Curr Pharm Des 9: 827-831.

Paxinos G, Watson C (1986). The rat brain stereotaxic coordinates. Academic Press: New York.

Phillis JW, O'Regan MH (2004). A potentially critical role of phospholipases in central nervous system ischemic, traumatic, and neurodegenerative disorders. Brain Res Brain Res Rev 44: 13-47.

Piazza PV, Barrot M, Rouge-Pont F, Marinelli M, Maccari S, Abrous DN et al (1996). Suppression of glucocorticoid secretion and antipsychotic drugs have similar effects on the mesolimbic dopaminergic transmission. Proc Natl Acad Sci USA 93: 15445-15450.

Rintala J, Seemann R, Chandrasekaran K, Rosenberger TA, Chang L, Contreras MA et al (1999). $85 \mathrm{kDa}$ cytosolic phospholipase A2 is a target for chronic lithium in rat brain. Neuroreport 10: 3887-3890.

Rosenberger TA, Villacreses NE, Hovda JT, Bosetti F, Weerasinghe G, Wine RN et al (2004). Rat brain arachidonic acid metabolism is increased by a 6-day intracerebral ventricular infusion of bacterial lipopolysaccharide. J Neurochem 88: 1168-1178.

Rouge-Pont F, Abrous DN, Le Moal M, Piazza PV (1999). Release of endogenous dopamine in cultured mesencephalic neurons: influence of dopaminergic agonists and glucocorticoid antagonists. Eur J Neurosci 11: 2343-2350.

Saija A, Princi P, Lanza M, Scalese M, Aramnejad E, De Sarro A (1995). Systemic cytokine administration can affect blood-brain barrier permeability in the rat. Life Sci 56: 775-784.

Schatzberg AF, Rothschild AJ, Langlais PJ, Bird ED, Cole JO (1985). A corticosteroid/dopamine hypothesis for depression and related states. J Psychiatr Res 19: 57-64.

Schwartz K, Yadid G, Weizman A, Rehavi M (2003). Decreased limbic vesicular monoamine transporter 2 in a genetic rat model of depression. Brain Res 965: 174-179.
Smith T, Hewson AK, Quarrie L, Leonard JP, Cuzner ML (1994). Hypothalamic PGE2 and cAMP production and adrenocortical activation following intraperitoneal endotoxin injection: in vivo microdialysis studies in Lewis and Fischer rats. Neuroendocrinology 59: 396-405.

Song C, Lin A, Bonaccorso S, Heide C, Verkerk R, Kenis G et al (1998). The inflammatory response system and the availability of plasma tryptophan in patients with primary sleep disorders and major depression. J Affect Disord 49: 211-219.

Song C, Merali Z, Anisman H (1999). Variations of nucleus accumbens dopamine and serotonin following systemic interleukin-1, interleukin-2 or interleukin-6 treatment. Neuroscience 88: 823-836.

Song C, Li X, Leonard BE, Horrobin DF (2003). Effects of dietary n-3 or n-6 fatty acids on interleukin-1beta-induced anxiety, stress, and inflammatory responses in rats. J Lipid Res 44: 1984-1991.

Song C, Horrobin D (2004). Omega-3 fatty acid ethyl-eicosapentaenoate, but not soybean oil, attenuates memory impairment induced by central IL-1beta administration. J Lipid Res 45: $1112-1121$.

Song C, Phillips TG, Leonard BE, Horrobin DF (2004). Ethyleicosapentaenoic acid ingestion prevents corticosteronemediated memory impairment induced by central administration of interleukin-1beta in rats. Mol Psychiatry 9: 630-638.

Strokin M, Sergeeva M, Reiser G (2003). Docosahexaenoic acid and arachidonic acid release in rat brain astrocytes is mediated by two separate isoforms of phospholipase A2 and is differently regulated by cyclic AMP and $\mathrm{Ca} 2+. \mathrm{Br} J$ Pharmacol 139: 1014-1022.

Sun GY, Xu J, Jensen MD, Simonyi A (2004). Phospholipase A2 in the central nervous system: implications for neurodegenerative diseases. J Lipid Res 45: 205-213.

Tappia PS, Man WJ, Grimble RF (1995). Influence of unsaturated fatty acids on the production of tumour necrosis factor and interleukin- 6 by rat peritoneal macrophages. Mol Cell Biochem 143: 89-98.

Tariq M, Khan HA, Al Moutaery K, Al Deeb S (2001). Protective effect of quinacrine on striatal dopamine levels in 6-OHDA and MPTP models of Parkinsonism in rodents. Brain Res Bull 54: 77-82.

Tully AM, Roche HM, Doyle R, Fallon C, Bruce I, Lawlor B et al (2003). Low serum cholesteryl ester-docosahexaenoic acid levels in Alzheimer's disease: a case-control study. Br J Nutr 89: 483-489.

van der Meer MJ, Sweep CG, Pesman GJ, Tilders FJ, Hermus AR (1996). Chronic stimulation of the hypothalamus-pituitaryadrenal axis in rats by interleukin 1beta: central and peripheral mechanisms. Cytokine 8: 910-919.

Wu YL, Yoshida M, Emoto H, Tanaka M (1999). Psychological stress selectively increases extracellular dopamine in the 'shell', but not in the 'core' of the rat nucleus accumbens: a novel dualneedle probe simultaneous microdialysis study. Neurosci Lett 275: 69-72.

Xu J, Yu S, Sun A, Sun G (2003). Oxidant-mediated AA release from astrocytes involves cPLA2 and iPLA2. Free Rad Biol Med 12: $1531-1543$.

Yokotani K, Wang M, Murakami Y, Okada S, Hirata M (2000). Brain phospholipase $\mathrm{A}(2)$-arachidonic acid cascade is involved in the activation of central sympatho-adrenomedullary outflow in rats. Eur J Pharmacol 398: 341-347.

Zhang J, Rivest S (2000). A functional analysis of EP4 receptor-expressing neurons in mediating the action of prostaglandin E2 within specific nuclei of the brain in response to circulating interleukin-1beta. J Neurochem 74 2134-2145. 